\title{
Existence of a positive solution for a singular system
}

\author{
Marcelo Montenegro \\ Universidade Estadual de Campinas, IMECC, \\ Departamento de Matemática, \\ Caixa Postal 6065 CEP 13083-970, Campinas, SP Brazil \\ E-mail: msm@ime.unicamp.br \\ Antonio Suárez \\ Dpto. Ecuaciones Diferenciales y Análisis Numérico \\ Fac. Matemáticas, C/ Tarfia s/n \\ C.P. 41012, Univ. Sevilla, Spain \\ E-mail: suarez@us.es
}

ABSTRACT. We show the existence and nonexistence of positive solutions to a system of singular elliptic equations with Dirichlet boundary condition. This system arises in studies of pattern formation in biology and in the activator-inhibitor model proposed by Gierer-Meinhardt.

AMS SubJeCt Classification 2000. 35J25, 35J55.

KEYWORDS. Singular equations, Gierer-Meinhardt model, Elliptic system, Sub-Supersolutions method

\section{Introduction}

In this paper we study the system

$$
\begin{cases}-\Delta u=\lambda u^{q_{1}}-\frac{u^{p_{1}}}{v^{\beta_{1}}} & \text { in } \Omega, \\ -\Delta v=\mu v^{q_{2}}-\frac{u^{p_{2}}}{v^{\beta_{2}}} & \text { in } \Omega, \\ u=v=0 & \text { on } \partial \Omega,\end{cases}
$$

where $\Omega \subset \mathbb{R}^{N}, N \geq 1$, is a bounded domain with smooth boundary $\partial \Omega$,

$$
\lambda, \mu \in \mathbb{R}, \quad 0<q_{1}, q_{2}, \beta_{1}, \beta_{2}<1 \quad \text { and } \quad p_{1}, p_{2}>0 .
$$


Our main goal in this paper is to show results about existence and nonexistence of positive solutions of (1.1) in terms of the parameters $\lambda$ and $\mu$. It is clear that, thanks to the maximum principle, if $\lambda \leq 0$ or $\mu \leq 0$ then (1.1) does not possess positive solutions. With respect to the existence, our main result is

Theorem 1.1. (A) Assume that $q_{1}<p_{1}$. There is a constant $\lambda^{*}(\Omega)>0$ depending on $\Omega$ such that for

$$
\mu \geq \lambda^{*}(\Omega) \lambda^{\sigma} \quad \text { and } \quad \lambda>0
$$

where

$$
\sigma=\frac{p_{2}\left(1-q_{2}\right)}{\left(1+\beta_{2}\right)\left(1-q_{1}\right)}
$$

there exists a positive $C^{1, \Upsilon}(\bar{\Omega}), 0<\Upsilon<1$ solution of (1.1).

(B) Assume that $q_{1} \geq p_{1}$. There is a constant $\lambda_{*}(\Omega)>0$ depending on $\Omega$ such that for

$$
\lambda<\lambda_{*}(\Omega) \mu^{-r} \quad \text { and } \quad \mu>0,
$$

where

$$
r=\frac{\beta_{1}\left(1-q_{1}\right)}{\left(1-p_{1}\right)\left(1-q_{2}\right)},
$$

then (1.1) does not possess a positive solution.

Systems of singular equations like (1.1) are the stationary counterpart of general evolutionary problems of the form

$$
\begin{cases}u_{t}=\eta \Delta u+\lambda u^{q_{1}}-\gamma \frac{u^{p_{1}}}{v^{\beta_{1}}} & \text { in } \Omega, \\ v_{t}=\delta \Delta v+\mu v^{q_{2}}-\theta \frac{u^{p_{2}}}{v^{\beta_{2}}} & \text { in } \Omega, \\ u=v=0 & \text { on } \partial \Omega .\end{cases}
$$

In the original model proposed by Gierer-Meinhardt [10],

$\eta, \delta>0, \quad \lambda, \mu, \gamma, \theta<0, \quad q_{1}=q_{2}=1, \quad p_{1}, p_{2}, \beta_{1}, \beta_{2}>0, \quad 0<\left(p_{1}-1\right) / \beta_{1}<p_{2} /\left(\beta_{2}+1\right)$

and the boundary conditions are of Neumann type. This system was motivated by biological experiments on hydra in morphogenesis, where $u$ represents the density of an activator chemical substance and $v$ is an inhibitor. The slow diffusion of $u$ and the fast diffusion of $v$ is translated into the fact that $\eta$ is small and $\delta$ is large, see also $[11,16,18]$ for an account on biological applications of such systems. There are a few papers dealing with scalar equations $[1,4,5,8,19]$ and references therein.

According to an observation made in [3], it is natural to study (1.3) with Dirichlet boundary conditions, since numerical experiments from [10] exhibit solutions approaching zero near the boundary of $\Omega$. Moreover, Neumann condition is not explicitly mentioned in the original paper [10]. Although, the majority of early papers deal with a system on a bounded domain with Neumann boundary conditions.

The stationary system with

$$
\eta=\delta=1, \quad \lambda=\mu=\gamma=\theta=-1 \quad \text { and } \quad p_{1}=p_{2}=q_{1}=q_{2}=\beta_{1}=\beta_{2}=1 .
$$


was studied in [2]. Thus for the system

$$
\begin{cases}-\Delta u=-u+\frac{u^{p_{1}}}{v^{\beta_{1}}} & \text { in } \Omega, \\ -\Delta v=-v+\frac{u^{p_{2}}}{v^{\beta_{2}}} & \text { in } \Omega, \\ u=v=0 & \text { on } \partial \Omega,\end{cases}
$$

they have shown existence and nonexistence of solutions and uniqueness of solution in one dimension. Another uniqueness result for (1.4) was proved in [3], in the situation

$$
\eta=\delta=1, \quad \lambda=\mu=\gamma=\theta=-1 \quad \text { and } \quad p_{1}=p_{2}>1, \quad \beta_{2}=0, \quad \beta_{1}=q_{1}=q_{2}=1 .
$$

A study allowing more general singular nonlinearities was performed in $[9,13,14]$.

We are interested in studying stationary states of (1.3) for a different range of parameters and constants (1.2). Notice that our results depend strongly on the size of $q_{1}$ and $p_{1}$. Indeed, in the existence part (A) of Theorem 1.1 we require $q_{1}<p_{1}$, and the conclusion holds for $\lambda>0$ and $\mu \geq C \lambda^{\sigma}$ for some positive constants $C$ and $\sigma$. Part (B) demands $q_{1} \geq p_{1}$, thus the nonexistence of solution is inferred for $\lambda>0$ and $\mu<C \lambda^{-r}$ for some positive constants $C$ and $r$. In order to obtain our main results we use an adequate sub-supersolution method, which will be detailed later.

The paper is organized as follows. In section 2 we show that the sub-supersolution method holds for our system, which has singular nonlinearities, generalizing classical results, see for instance [17]. In section 3 we study some auxiliary problems related to sublinear equations, singular equations and porous medium logistic equation. Section 4 is devoted to the proof of Theorem 1.1.

\section{The sub-super method for singular systems}

First of all we show that the sub-supersolution method works well for singular systems. We consider the general system

$$
\begin{cases}-\Delta u=f(x, u, v) & \text { in } \Omega, \\ -\Delta v=g(x, u, v) & \text { in } \Omega, \\ u=v=0 & \text { on } \partial \Omega,\end{cases}
$$

where $f, g: \Omega \times \mathbb{R} \times \mathbb{R} \mapsto \mathbb{R}$ are Caratheodory functions. On the other hand, we denote by

$$
\rho_{0}(x)=\operatorname{dist}(x, \partial \Omega)
$$

and given $w \leq z$ a.e. in $\Omega$

$$
[w, z]:=\{u: w(x) \leq u(x) \leq z(x) \text { a.e. } x \in \Omega\} .
$$

The notions of solutions and sub-supersolutions of (2.1) are:

Definition 2.1. We say that $(u, v) \in\left(L^{1}(\Omega)\right)^{2}$ is a solution of (2.1) if

1. $f(\cdot, u, v) \rho_{0}, g(\cdot, u, v) \rho_{0} \in L^{1}(\Omega)$; 
2.

$$
-\int_{\Omega} u \Delta \xi=\int_{\Omega} f(x, u, v) \xi, \quad-\int_{\Omega} v \Delta \xi=\int_{\Omega} g(x, u, v) \xi, \quad \forall \xi \in C_{0}^{2}(\bar{\Omega}) .
$$

Definition 2.2. We say that $(\underline{u}, \underline{v}),(\bar{u}, \bar{v}) \in\left(L^{1}(\Omega)\right)^{2}$ is a pair of sub-supersolutions of (2.1) if

1. $\underline{u} \leq \bar{u}$ and $\underline{v} \leq \bar{v}$ in $\Omega$;

2.

$$
\begin{aligned}
& f(\cdot, u, v) \rho_{0}, f(\cdot, u, v) \rho_{0} \in L^{1}(\Omega) \quad \text { for all } u \in[\underline{u}, \bar{u}] \text { and } v \in[\underline{v}, \bar{v}], \\
& g(\cdot, u, v) \rho_{0}, g(\cdot, u, v) \rho_{0} \in L^{1}(\Omega) \quad \text { for all } u \in[\underline{u}, \bar{u}] \text { and } v \in[\underline{v}, \bar{v}]
\end{aligned}
$$

3. for all $\xi \in C_{0}^{2}(\bar{\Omega}), \xi \geq 0$,

$$
-\int_{\Omega} \underline{u} \Delta \xi-\int_{\Omega} f(x, \underline{u}, v) \xi \leq 0 \leq-\int_{\Omega} \bar{u} \Delta \xi-\int_{\Omega} f(x, \bar{u}, v) \xi, \quad \forall v \in[\underline{v}, \bar{v}] ;
$$

and

$$
-\int_{\Omega} \underline{v} \Delta \xi-\int_{\Omega} g(x, u, \underline{v}) \xi \leq 0 \leq-\int_{\Omega} \bar{v} \Delta \xi-\int_{\Omega} g(x, u, \bar{v}) \xi, \quad \forall u \in[\underline{u}, \bar{u}] .
$$

Next we prove that the existence of a pair of sub-supersolutions implies the existence of a solution of the system.

Theorem 2.3. Assume that there exists a pair of sub-supersolution $(\underline{u}, \underline{v}),(\bar{u}, \bar{v})$ of (2.1). Then, there exists a solution $(u, v)$ of (2.1) such that $\underline{u} \leq u \leq \bar{u}$ and $\underline{v} \leq v \leq \bar{v}$ in $\Omega$.

Proof. First, we define the truncations

$$
T u(x):= \begin{cases}\bar{u}(x) & \text { if } u(x) \geq \bar{u}(x), \\ u(x) & \text { if } \underline{u}(x) \leq u(x) \leq \bar{u}(x), \\ \underline{u}(x) & \text { if } u(x) \leq \underline{u}(x),\end{cases}
$$

and

$$
S v(x):= \begin{cases}\bar{v}(x) & \text { if } v(x) \geq \bar{v}(x), \\ v(x) & \text { if } \underline{v}(x) \leq v(x) \leq \bar{v}(x), \\ \underline{v}(x) & \text { if } v(x) \leq \underline{v}(x) .\end{cases}
$$

We denote by

$$
L^{1}\left(\rho_{0}, \Omega\right):=\left\{u: u \rho_{0} \in L^{1}(\Omega)\right\} .
$$

We define the Nemytskii operators (well defined by (2.2))

$$
\begin{aligned}
F: L^{1}(\Omega) \times L^{1}(\Omega) & \mapsto L^{1}\left(\rho_{0}, \Omega\right) \\
(u, v) & \mapsto F(u, v):=f(x, T u, S v)
\end{aligned}
$$


and similarly

$$
\begin{aligned}
G: L^{1}(\Omega) \times L^{1}(\Omega) & \mapsto L^{1}\left(\rho_{0}, \Omega\right) \\
(u, v) & \mapsto G(u, v):=g(x, T u, S v) .
\end{aligned}
$$

We define the operator $K: L^{1}\left(\rho_{0}, \Omega\right) \mapsto L^{1}(\Omega)$ by $h \mapsto w:=K(h)$, being $w$ the unique solution of

$$
\begin{cases}-\Delta w=h & \text { in } \Omega, \\ w=0 & \text { on } \partial \Omega .\end{cases}
$$

It can be proved:

1. $F$ and $G$ are continuous (Theorem 2.1 in [15], the notion of equi-integrability is not needed here).

2. $[F, G]\left(L^{1}(\Omega)\right)^{2}$ is bounded in $L^{1}\left(\rho_{0}, \Omega\right)$, since $T$ and $S$ defined by (2.3) and (2.4) are bounded.

3. $K \circ F$ and $K \circ G$ are continuos and compact operators from $\left(L^{1}(\Omega)\right)^{2}$ to $L^{1}(\Omega)$ (Theorem 3.1 in [15]).

Then, by the Schauder's fixed point theorem, we can conclude the existence of a solution $(u, v) \in\left(L^{1}(\Omega)\right)^{2}$ of

$$
\begin{cases}-\Delta u=f(x, T u, S v) & \text { in } \Omega, \\ -\Delta v=g(x, T u, S v) & \text { in } \Omega, \\ u=v=0 & \text { on } \partial \Omega .\end{cases}
$$

We claim that $(u, v) \in[\underline{u}, \bar{u}] \times[\underline{v}, \bar{v}]$ and so $(u, v)$ is solution of $(2.1)$. Indeed, let

$$
w:=u-\bar{u}
$$

Then, for all $V \in[\underline{v}, \bar{v}]$ and all $\xi \in C_{0}^{2}(\bar{\Omega}), \xi \geq 0$, we get

$$
-\int_{\Omega} w \Delta \xi \leq \int_{\Omega}(f(x, T u, S v)-f(x, \bar{u}, V)) \xi
$$

and then taking $V=S v$

$$
-\int_{\Omega} w \Delta \xi \leq \int_{\Omega}(f(x, T u, S v)-f(x, \bar{u}, S v)) \xi .
$$

Then, applying the Kato's inequality (see Proposition 3.1 in [15]) we obtain

$$
-\int_{\Omega} w^{+} \Delta \xi \leq \int_{[u \geq \bar{u}]}(f(x, T u, S v)-f(x, \bar{u}, S v)) \xi=0 \quad \forall \xi \in C_{0}^{2}(\bar{\Omega}), \xi \geq 0 .
$$

We deduce that $w^{+}=0$ a.e.; and conclude the proof.

Remark 2.4. Assuming more regularity to $f, g$ and the pair of sub-supersolution, we can obtain that the solution lies in a better space, see Section 5 in [15]. See also Remark 3.6. 


\section{Some auxiliary problems}

In order to find a pair of sub-supersolutions of (1.1) we need to study some scalar equations. First of all, given $\lambda \in \mathbb{R}$ and $0<q<1$, consider

$$
\begin{cases}-\Delta u=\lambda u^{q} & \text { in } \Omega, \\ u=0 & \text { on } \partial \Omega .\end{cases}
$$

It is well-known that there exists a unique positive solution of (3.1) if, and only if, $\lambda>0$. We denote this solution by $\omega_{[\lambda, q]}$; moreover

$$
\omega_{[\lambda, q]}=\lambda^{1 /(1-q)} \omega_{[1, q]} .
$$

It is known that there exist constants $k$ and $K$ with $0<k<K<+\infty$ such that

$$
k \rho_{0}(x) \leq \omega_{[\lambda, q]}(x) \leq K \rho_{0}(x) \quad x \in \Omega .
$$

We need to study the following problem

$$
\begin{cases}-\Delta u=\lambda f(x, u)-\frac{a(x)}{u^{\beta}} & \text { in } \Omega, \\ u=0 & \text { on } \partial \Omega\end{cases}
$$

where $\beta \in(0,1)$ and

$$
a: \Omega \rightarrow \mathbb{R} \quad \text { is a continuous positive function, }
$$

$$
\text { there is } 1<\gamma<2 \quad \text { such that } \quad \limsup _{x \rightarrow \partial \Omega} \frac{a(x)}{\rho_{0}(x)^{\gamma(1+\beta)-2}}<+\infty \text {, }
$$

$$
\begin{aligned}
& f: \Omega \times \mathbb{R} \rightarrow \mathbb{R} \text { is a continuous function, } \\
& f(x, s)>0 \quad \text { for } s \neq 0, \\
& \lim _{s \rightarrow+\infty} \frac{f(x, s)}{s}=0 \quad \text { uniformly in } x .
\end{aligned}
$$

In the following result we characterize the existence of positive solution of (3.3).

Proposition 3.1. There exists $\lambda^{*} \in(0,+\infty)$ such that for all $\lambda \geq \lambda^{*}$, problem (3.3) has a positive a.e. weak solution and no positive solution for $\lambda<\lambda^{*}$.

Proof. We are going to apply the sub-supersolution method from [15]. Take

$$
\underline{u}:=c \varphi_{1}^{\gamma}, \quad \bar{u}:=K e,
$$

for $c, K>0$ such that $\underline{u} \leq \bar{u}$ in $\Omega$, where $e$ is the unique positive solution of

$$
\begin{cases}-\Delta e=1 & \text { in } \Omega, \\ e=0 & \text { on } \partial \Omega\end{cases}
$$


and $\varphi_{1}>0$ is the first eigenfunction of the Laplacian in $H_{0}^{1}(\Omega)$ such that $\left\|\varphi_{1}\right\|_{\infty}=1$. Recall that there exist positive constants $0<c<C<\infty$ such that

$$
0<c \rho_{0}(x) \leq e(x), \varphi_{1}(x) \leq C \rho_{0}(x), \quad \forall x \in \Omega .
$$

First, observe that

$$
\left|\lambda f(x, u)-\frac{a(x)}{u^{\beta}}\right| \rho_{0} \in L^{1}(\Omega), \quad \forall u \in[\underline{u}, \bar{u}] .
$$

Indeed, for $u \in[\underline{u}, \bar{u}]$ we have

$$
\left|a(x) u^{-\beta}\right| \rho_{0} \leq C a(x) \rho_{0}^{-\gamma \beta+1} \leq C \rho_{0}^{\gamma-1} \in L^{1}(\Omega)
$$

if $\gamma-1>-1$.

To show that $\underline{u}$ is subsolution, we need to verify

$$
-\Delta \underline{u}+\frac{a(x)}{\underline{u}^{\beta}}=-c \gamma(\gamma-1) \varphi_{1}^{\gamma-2}\left|\nabla \varphi_{1}\right|^{2}+c \gamma \lambda_{1} \varphi_{1}^{\gamma}+a(x) c^{-\beta} \varphi_{1}^{-\beta \gamma} \leq \lambda f\left(x, c \varphi_{1}^{\gamma}\right) \quad \text { in } \Omega .
$$

We distinguish two cases:

(i) Near the boundary $\partial \Omega$ :

For every $M>0$ there is a $\delta>0$ such that for every

$$
x \in \Omega_{\delta}:=\left\{x \in \Omega: \rho_{0}(x)<\delta\right\}
$$

one has by (3.5)

$$
\begin{gathered}
-c \gamma(\gamma-1) \varphi_{1}^{\gamma-2}\left|\nabla \varphi_{1}\right|^{2}+a(x) c^{-\beta} \varphi_{1}^{-\beta \gamma}=c^{-\beta} \varphi_{1}^{\gamma-2}\left[-c^{1+\beta} \gamma(\gamma-1)\left|\nabla \varphi_{1}\right|^{2}+\frac{a(x)}{\varphi_{1}^{\gamma-2+\beta \gamma}}\right] \\
\leq c^{-\beta} \varphi_{1}^{\gamma-2}\left[-c^{1+\beta} \gamma(\gamma-1)\left|\nabla \varphi_{1}\right|^{2}+M\right] \leq \frac{-c}{2} \gamma(\gamma-1) \varphi_{1}^{\gamma-2}\left|\nabla \varphi_{1}\right|^{2}
\end{gathered}
$$

for a sufficiently large $c>0$.

In this way, taking $\delta$ smaller if necessary, we get

$$
-\Delta \underline{u}+\frac{a(x)}{\underline{u}^{\beta}} \leq c \gamma \varphi_{1}^{\gamma-2}\left[-\frac{(\gamma-1)}{2}\left|\nabla \varphi_{1}\right|^{2}+\lambda_{1} \varphi_{1}^{2}\right] \leq 0 .
$$

Notice that if $M=0$, we can take $c>0$ arbitrary.

(ii) Inner points $x \in \Omega \backslash \bar{\Omega}_{\delta}$.

Once $c$ has been fixed above, take $\lambda$ large enough in such a way that

$$
c^{1+\beta} \gamma \lambda_{1} \varphi_{1}^{\gamma}+a(x) \varphi_{1}^{-\beta \gamma} \leq \lambda c^{\beta} f\left(x, c \varphi_{1}^{\gamma}\right) .
$$

On the other hand, with respect to the supersolution we need that

$$
-\Delta \bar{u} \geq \lambda f(x, \bar{u})-\frac{a(x)}{\bar{u}^{\beta}},
$$

for which it suffices that

$$
K \geq \lambda f(x, K e)
$$


This is promptly verified for $K$ large enough thanks to (3.8).

We claim that there is no positive solution of (3.3) if $\lambda>0$ is small. Indeed, if $u>0$ is an existing solution, multiply the equation by $\varphi_{1}$ and integrate. Hence,

$$
\int_{\Omega}\left(\lambda_{1} \varphi_{1} u+\frac{a(x)}{u^{\beta}} \varphi_{1}\right)=\lambda \int_{\Omega} f(x, u) \varphi_{1}
$$

Let $\delta>0$ and $\Omega^{\delta}:=\left\{x \in \Omega: \rho_{0}(x)>\delta\right\}$. Thus

$$
c \int_{\Omega^{\delta}}\left(u+\frac{1}{u^{\beta}}\right) \varphi_{1} \leq \int_{\Omega^{\delta}}\left(\lambda_{1} u+\frac{a(x)}{u^{\beta}}\right) \varphi_{1}<\lambda \int_{\Omega} f(x, u) \varphi_{1}
$$

where $c$ is a constant depending on $\delta, \Omega$ and $\|a\|_{L^{\infty}\left(\Omega^{\delta}\right)}$. Since

$$
\lambda \int_{\Omega} f(x, u) \varphi_{1} \rightarrow 0 \quad \text { as } \lambda \rightarrow 0
$$

we get a contradiction since $u+1 / u^{\beta}$ is bounded from below and $\int_{\Omega} f(x, u) \varphi_{1}$ is bounded. This last assertion follows from the fact that $u$ is a priori bounded independently from $\lambda$ by a bootstrap argument, since there is a constant $C>0$ such that $-\Delta u \leq C \lambda(1+u)$ for every $u$.

Setting

$$
\lambda^{*}=\inf \{\lambda>0 \mid \text { such that (3.3) has a positive a.e. solution }\} .
$$

Then $\lambda^{*}<+\infty$ and for all $\lambda \geq \lambda^{*}$, problem (3.3) has a positive a.e. weak solution.

Remark 3.2. If $\gamma-2+\beta \gamma>0$, then in view of (3.5), $a(x) \rightarrow 0$ as $x \rightarrow \partial \Omega$. This is true if $\beta \geq 1$ for example.

If $\gamma-2+\beta \gamma<0$, then eventually $0<\beta<1$ and $a(x) \rightarrow 0$ as $x \rightarrow \partial \Omega$ or $a(x) \rightarrow+\infty$ as $x \rightarrow \partial \Omega$. But with (3.5) satisfied.

We now consider a particular case of (3.3),

$$
\begin{cases}-\Delta u=\lambda u^{q}-a(x) \frac{1}{u^{\beta}} & \text { in } \Omega, \\ u=0 & \text { on } \partial \Omega,\end{cases}
$$

where $0<q, \beta<1$ and $a$ verifies (3.4) and (3.5).

Proposition 3.3. There exists $\lambda^{*}(a)>0$ such that a positive maximal solution of (3.11) exists if, and only if,

$$
\lambda \geq \lambda^{*}(a) .
$$

We denote this maximal solution by $\Theta_{[\lambda, q, \beta, a]}$. Moreover, the map $a \mapsto \lambda^{*}(a)$ is increasing. Furthermore, if $a \in C(\bar{\Omega})$, there exist constants $c$ and $C$ such that

$$
c \rho_{0}(x) \leq \Theta_{[\lambda, q, \beta, a]}(x) \leq C \rho_{0}(x) .
$$

Proof. The existence of a positive solution as well as $\lambda^{*}(a)$ follow by Proposition 3.1. The maximality of the solution is due to the fact that any positive solution of (3.3) is a subsolution of (3.1).

The fact that $a \mapsto \lambda^{*}(a)$ is increasing is immediate.

The existence of the constant $c$ verifying (3.12) is due to the Hopf maximum principle and $C$ is due to the $C^{1}(\bar{\Omega})$ regularity of the solution, see also Remark 3.6. 
We need some properties of the porous medium logistic equation with a possibly singular weight

$$
\begin{cases}-\Delta u=\lambda u^{q}-N(x) u^{p} & \text { in } \Omega, \\ u=0 & \text { on } \partial \Omega,\end{cases}
$$

where $0<q<1, p>0$ with

$$
0<N \leq k \rho_{0}(x)^{\beta}, \quad k>0,
$$

$N \in C(\Omega)$ and $\beta \in \mathbb{R}$ (possibly negative).

Proposition 3.4. Assume that $\beta+p>-1$.

1. If $q<p$, then there exists a unique $C^{1}(\bar{\Omega})$ positive solution if, and only if, $\lambda>0$.

2. If $q \geq p$, then there exists $\lambda_{*}(N) \geq 0$ such that there exists a positive $C^{1}(\bar{\Omega})$ solution if, and only if, $\lambda \geq \lambda_{*}(N)$.

Moreover, if $N \geq N_{0}>0$ for some $N_{0} \in \mathbb{R}$ then $\lambda_{*}(N)>0$.

Proof. Take $\bar{u}:=K e$ and $\underline{u}:=\varepsilon \varphi_{1}^{r}, r \geq 1$ and $K, \varepsilon>0$ positive constants to be chosen later. In order to apply the sub-supersolution method we need that

$$
\left|N(x) u^{p}\right| \rho_{0} \in L^{1}(\Omega), \quad \forall u \in[\underline{u}, \bar{u}] .
$$

Observe that (3.14) implies

$$
\left|N(x) u^{p}\right| \rho_{0} \leq K \rho_{0}^{\beta+p+1}
$$

and so $\left|N(x) u^{p}\right| \rho_{0} \in L^{1}(\Omega)$ if

$$
\beta+p>-2 .
$$

First observe that $\underline{u}$ is subsolution of (3.13) provided that

$$
r(1-r) \varepsilon^{1-q} \varphi_{1}^{r(1-q)-2}\left|\nabla \varphi_{1}\right|^{2}+r \varepsilon^{1-q} \lambda_{1} \varphi_{1}^{r(1-q)}+C \varepsilon^{p-q} \varphi_{1}^{r(p-q)+\beta} \leq \lambda .
$$

On the other hand, $\bar{u}$ is supersolution if $K$ is taken large. Take also $K$ large such that $\underline{u} \leq \bar{u}$ in $\Omega$. So, it suffices to verify (3.15). For that, we consider two cases:

1. Assume that $p>q$. Take $r>1$ such that $r(p-q)+\beta>0$. Then, recalling that $\left\|\varphi_{1}\right\|_{\infty}=1,(3.15)$ is satisfied if

$$
r \varepsilon^{1-q} \lambda_{1}+C \varepsilon^{p-q} \leq \lambda
$$

for which it suffices to take $\varepsilon$ sufficiently small.

With respect to the uniqueness, the result follows applying Theorem 2.1 in [6], specifically taking $g(t)=t^{q}$.

2. Assume now that $p \leq q$. Take now $\varepsilon=1$. Again we distinguish two cases:

(i) Near the boundary $\partial \Omega$ :

Take in this case $r \geq 1$ and $r(1-q)-2<r(p-q)+\beta$, or equivalently, $r(1-p)<\beta+2$. Then we need that $1<(2+\beta) /(1-p)$ or equivalently $-1<\beta+p$. In this case, (3.15) is equivalent to

$$
\varphi_{1}^{r(1-q)-2}\left[r(1-r)\left|\nabla \varphi_{1}\right|^{2}+r \lambda_{1} \varphi_{1}^{2}+C \varphi_{1}^{r(p-1)+\beta+2}\right] \leq \lambda .
$$


Take $\delta>0$ small enough such that

$$
r(1-r)\left|\nabla \varphi_{1}\right|^{2}+r \lambda_{1} \varphi_{1}^{2}+C \varphi_{1}^{r(p-1)+\beta+2}<0
$$

in $\Omega_{\delta}=\left\{x \in \Omega: \rho_{0}(x)<\delta\right\}$.

(ii) Inner points:

In the region $\Omega \backslash \bar{\Omega}_{\delta}$ we have that $\varphi_{1} \geq c(\delta)$ for some $c(\delta)>0$. Hence, for (3.15) it is sufficient that

$$
r \lambda_{1}+C(\delta) \leq \lambda
$$

for some $C(\delta)$. Fixed $\delta$, we can take $\lambda$ large.

Hence, we can define

$$
\lambda_{*}(N)=\inf \{\lambda>0 \mid \text { such that (3.13) has a positive a.e. solution }\} .
$$

Then $\lambda_{*}(N)<+\infty$ and for all $\lambda \geq \lambda_{*}(N)$, problem (3.13) has a positive a.e. weak solution.

Finally, assume that $N \geq N_{0}>0$ and $q \geq p$. Then, multiplying the equation by $\varphi_{1}$ and integrating we have

$$
0=\int_{\Omega} \varphi_{1} u^{p}\left(\lambda u^{q-p}-N-\lambda_{1} u^{1-p}\right) \leq \int_{\Omega} \varphi_{1} u^{p}\left(\lambda u^{q-p}-N_{0}-\lambda_{1} u^{1-p}\right) .
$$

Assuming $q>p$, the maximum of the function $f(x):=\lambda x^{q-p}-\lambda_{1} x^{1-p}$ is attained at

$$
x_{M}=\left(\frac{\lambda(q-p)}{\lambda_{1}(1-p)}\right)^{1 /(1-q)}
$$

and

$$
f\left(x_{M}\right)=\lambda^{(1-p) /(1-q)}\left(\frac{q-p}{\lambda_{1}(1-p)}\right)^{(q-p) /(1-q)} \frac{1-q}{1-p}
$$

and so if $\lambda$ is small we have that

$$
\int_{\Omega} \varphi_{1} u^{p}\left(\lambda u^{q-p}-N_{0}-\lambda_{1} u^{1-p}\right)<0
$$

a contradiction. A similar argument can be used in the case $q=p$. This completes the proof.

Remark 3.5. Equations (3.3) and (3.11) have been studied in [5] and [19], but with different behavior of $a(x)$ or without $a(x)$. Also, equation (3.13) has been previously studied when $N$ is bounded, see [7] and references therein.

Remark 3.6. The solutions of Propositions 3.3, 3.4 and Theorem 1.1 (A) belong to $C^{1, \Upsilon}(\bar{\Omega}), 0<\Upsilon<1$. This follows from the results in [12] which says that if $-\Delta u=h$ in $\Omega$ with $u=0$ on $\partial \Omega$ and $\sup _{\bar{\Omega}}|h(x)| \rho_{0}^{\Upsilon}(x)<\infty$ for some $0<\Upsilon<1$, then $u \in C^{1,1-\Upsilon}(\bar{\Omega})$. 


\section{Proof of Theorem 1.1}

We are going to apply the sub-supersolution method to system (1.1). If we denote

$$
f(u, v):=\lambda u^{q_{1}}-\frac{u^{p_{1}}}{v^{\beta_{1}}} \quad g(u, v):=\mu v^{q_{2}}-\frac{u^{p_{2}}}{v^{\beta_{2}}},
$$

the third paragraph of the definition of sub-supersolution (Definition 2.2) is equivalent to

$$
-\Delta \underline{u} \leq f(\underline{u}, \underline{v}), \quad-\Delta \bar{u} \geq f(\bar{u}, \bar{v}),
$$

and

$$
-\Delta \underline{v} \leq g(\bar{u}, \underline{v}), \quad-\Delta \bar{v} \geq g(\underline{u}, \bar{v}) .
$$

We start the proof of Theorem 1.1:

Proof. (A) Take

$$
\bar{u}:=\omega_{\left[\lambda, q_{1}\right]}, \quad \text { and } \quad \bar{v}:=\omega_{\left[\mu, q_{2}\right]} .
$$

A subsolution is

$$
\underline{v}:=\Theta_{\left[\mu, q_{2}, \beta_{2}, \omega_{\left[\lambda, q_{1}\right]}^{p_{2}}\right]} .
$$

Observe that $\omega_{\left[\lambda, q_{1}\right]}=\lambda^{1 /\left(1-q_{1}\right)} \omega_{\left[1, q_{1}\right]}$ and so $\underline{v}$ verifies

$$
-\Delta v=\mu v^{q_{2}}-\lambda^{p_{2} /\left(1-q_{1}\right)} \frac{\omega_{\left[1, q_{1}\right]}^{p_{2}}}{v^{\beta_{2}}} \text { in } \Omega .
$$

Under the change of variable

$$
V=R v
$$

where

$$
R=\frac{1}{\lambda^{p_{2} /\left(\left(1-q_{1}\right)\left(1+\beta_{2}\right)\right)}},
$$

(4.3) transforms into

$$
\begin{cases}-\Delta V=\Lambda V^{q_{2}}-\frac{\omega_{\left[1, q_{1}\right]}^{p_{2}}}{V^{\beta_{2}}} & \text { in } \Omega \\ V=0 & \text { on } \partial \Omega\end{cases}
$$

where

$$
\Lambda=\mu \lambda^{-\sigma},
$$

with

$$
\sigma=\frac{p_{2}\left(1-q_{2}\right)}{\left(1-q_{1}\right)\left(1+\beta_{2}\right)}
$$

Observe that (4.4) is in the setting of (3.11) by taking $a=\omega_{\left[1, q_{1}\right]}^{p_{2}}$. Indeed, (3.4) and (3.5) are verified for all $\gamma$ such that

$$
\gamma \leq \frac{p_{2}+2}{1+\beta_{2}}
$$

which can be chosen $1<\gamma$. Hence, applying Proposition 3.3, we conclude the existence of a positive solution of (4.4) if

$$
\Lambda \geq \lambda^{*}(\Omega)
$$


or equivalently,

$$
\mu \geq \lambda^{*}(\Omega) \lambda^{\sigma}
$$

It is clear that $\underline{v} \leq \bar{v}$ and $\underline{v}>0$ if $\mu \geq \lambda^{*}(\Omega) \lambda^{\sigma}$. It remains to check that there exists $\underline{u}>0$ and satisfies

$$
-\Delta \underline{u} \leq \lambda \underline{u}^{q_{1}}-\underline{v}^{-\beta_{1}} \underline{u}^{p_{1}} \quad \text { in } \Omega \text {. }
$$

Let $u$ be the solution of

$$
\begin{cases}-\Delta u=\lambda u^{q_{1}}-\underline{v}^{-\beta_{1}} u^{p_{1}} & \text { in } \Omega, \\ u=0 & \text { on } \partial \Omega .\end{cases}
$$

Observe that in this case $N(x)=\underline{v}^{-\beta_{1}}$, being $\underline{v}$ defined in (4.2). Hence, taking into account (3.12) we obtain that $0<N \leq C \rho_{0}^{-\beta_{1}}$ and so it is clear that

$$
-\beta_{1}+p_{1}>-1
$$

Thus we can apply Proposition 3.4 to conclude that, if $q_{1}<p_{1}$, there exists a positive solution of (4.5) provided $\lambda>0$. Moreover, it is clear that $\underline{u} \leq \bar{u}$.

Finally, the second paragraph of Definition 2.2 is easy to verify.

In conclusion, if $q_{1}<p_{1}$ there is a positive solution of (1.1) if $\lambda>0$ and $\mu \geq \lambda^{*}(\Omega) \lambda^{\sigma}$. (B) Finally, we assume that $q_{1} \geq p_{1}$. Observe that if $(u, v)$ is a solution of (1.1), then

$$
v \leq \omega_{\left[\mu, q_{2}\right]}=\mu^{1 /\left(1-q_{2}\right)} \omega_{\left[1, q_{2}\right]}
$$

and then,

$$
-\Delta u \leq \lambda u^{q_{1}}-\mu^{-\beta_{1} /\left(1-q_{2}\right)} \omega_{\left[1, q_{2}\right]}^{-\beta_{1}} u^{p_{1}} .
$$

Under the change of variable

$$
U=R u, \quad R=\mu^{\beta_{1} /\left(\left(1-q_{2}\right)\left(1-p_{1}\right)\right)}
$$

the above inequality is transformed into

$$
\begin{cases}-\Delta U \leq \lambda \mu^{r} U^{q_{1}}-\omega_{\left[1, q_{2}\right]}^{-\beta_{1}} U^{p_{1}} & \text { in } \Omega \\ U=0 & \text { on } \partial \Omega\end{cases}
$$

Hence, multiplying by $\varphi_{1}$, integrating and with a similar argument to the proof of Proposition 3.4, we can conclude that if

$$
\lambda \mu^{r}<\lambda_{*}(\Omega)
$$

there is no positive solution of (1.1).

Acknowledgement. M. Montenegro was supported by CNPq-Brazil and A. Suárez was supported by MEC under grants MTM2006-07932. 


\section{References}

[1] Y. S. Choi, A. C. Lazer and P.J. McKenna, Some remarks on a singular elliptic boundary value problem, Nonlinear Anal. 32 (1998), 305-314.

[2] Y. S. Choi and P. J. McKenna, A singular Gierer-Meinhardt system of elliptic equations, Ann. Inst. Henri Poincaré, Analyse non linèaire 17 (2000), 503-522.

[3] Y. S. Choi and P. J. McKenna, A singular Gierer-Meinhardt system of elliptic equations: the classical case, Nonlinear Anal. 55 (2003), 521-541.

[4] M. G. Crandall, P. H. Rabinowitz and L. Tartar, On a Dirichlet problem with a singular nonlinearity, Comm. Partial Differential Equations 2 (1977), 193-222.

[5] J. Dávila and M. Montenegro, Positive versus free boundary solutions to a singular elliptic equation, J. Anal. Math., 90 (2003), 303-335.

[6] M. Delgado and A. Suárez, On the uniqueness of positive solution of an elliptic equation, Applied Mathematics Letters 18 (2005), 10891093.

[7] M. Delgado and A. Suárez, On the structure of the positive solutions of the logistic equation with nonlinear diffusion, J. Math. Anal. Appl. 268 (2002) 200-216.

[8] J. I. Díaz, J. M. Morel and L. Oswald, An elliptic equation with singular nonlinearity, Comm. Partial Differential Equations 12 (1987), 1333-1344.

[9] M. Ghergu and V. Radulescu, On a class of singular Gierer-Meinhardt systems arising in morphogenesis, C. R. Acad. Sci. Paris, Ser. I 344 (2007), 163-168.

[10] A. Gierer and H. Meinhardt, A theory of biological pattern formation, Kyberntik 12 (1972), 30-39.

[11] A. Gierer and H. Meinhardt, Generation and regeneration of sequence of structures during morphogenesis, J. Theoret. Biol. 85 (1980), 429-450.

[12] C. Gui and F. H. Lin, Regularity of an elliptic problem with a singular nonlinearity, Proc. Roy. Soc. Edinburgh Sect. A 123 (1993), 1021-1029.

[13] E. H. Kim, A class of singular Gierer-Meinhardt systems of elliptic boundary value problems, Nonlinear Anal. 59 (2004), 305-318.

[14] E. H. Kim, Singular Gierer-Meinhardt systems of elliptic boundary value problems, J. Math. Anal. Appl. 308 (2005), 1-10.

[15] M. Montenegro and A. Ponce, The sub-supersolution method for weak solutions, Proc. Amer. Math. Soc. 136 (2008), 2429-2438.

[16] W. M. Ni, Diffusion, cross-diffusion, and their spike-layer steady states, Notices Amer. Math. Soc. 45 (1998), 9-18.

[17] C. V. Pao, Nonlinear Parabolic and Elliptic Equations, Plenum Press, New York, 1992. 
[18] W. M. Ni, K. Suzuki and I. Takagi, The dynamics of a kinetics activator-inhibitor system, J. Differential Equations 229 (2006), 426-465.

[19] J. Shi and M. Yao, On a singular nonlinear semilinear elliptic problem, Proc. Roy. Soc. Edinburgh Sect. A 128 (1998), 1389-1401. 